Supplementary Information

\title{
Ultraslow Relaxation in Aprotic Double Salt Ionic Liquids
}

Adhip Rahman ${ }^{\dagger}$, M. Muhibur Rahman ${ }^{\dagger}$, M. Yousuf A. Mollah ${ }^{\ddagger}$ Md. Abu Bin Hasan Susan ${ }^{\dagger *}$ 


\section{S.1. Time-dependent Normalized Intensity Correlation Functions (ICFs) of the DSILs}

Figure S1 (a) - S1 (c) show the ICFs taken at $298 \mathrm{~K}$ for all of three DSILs. Data were taken starting from the first day after the preparation and each of the experiments was repeated one to two days afterwards. No pretreatment (for example - ultrasonicating) was performed before any of the experiments.
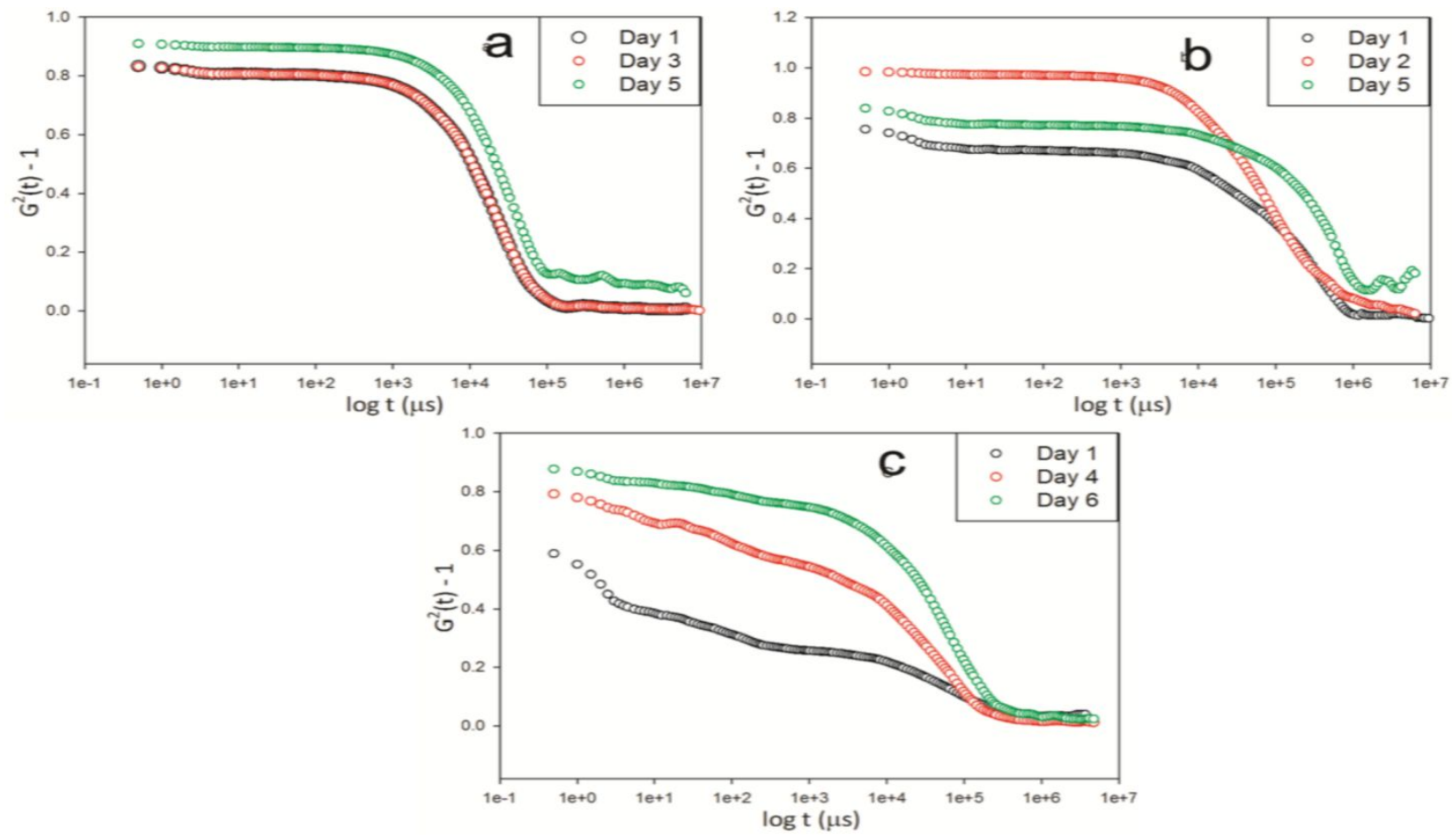

Figure S1. Time-dependent Normalized $\mathrm{G}^{2}(\mathrm{t})-1$ intensity correlation functions (ICFs) of (a) $\left[\mathrm{C}_{2} \mathrm{mim}\right][\mathrm{OTf}]_{0.5}\left[\mathrm{NTf}_{2}\right]_{0.5}$ (b) $\left[\mathrm{C}_{2} \mathrm{mim}\right]\left[\mathrm{BF}_{4}\right]_{0.5}\left[\mathrm{NTf}_{2}\right]_{0.5}$ and (c) $\left[\mathrm{C}_{2} \mathrm{mim}\right]\left[\mathrm{MeSO}_{3}\right]_{0.5}\left[\mathrm{NTf}_{2}\right]_{0.5}$ 

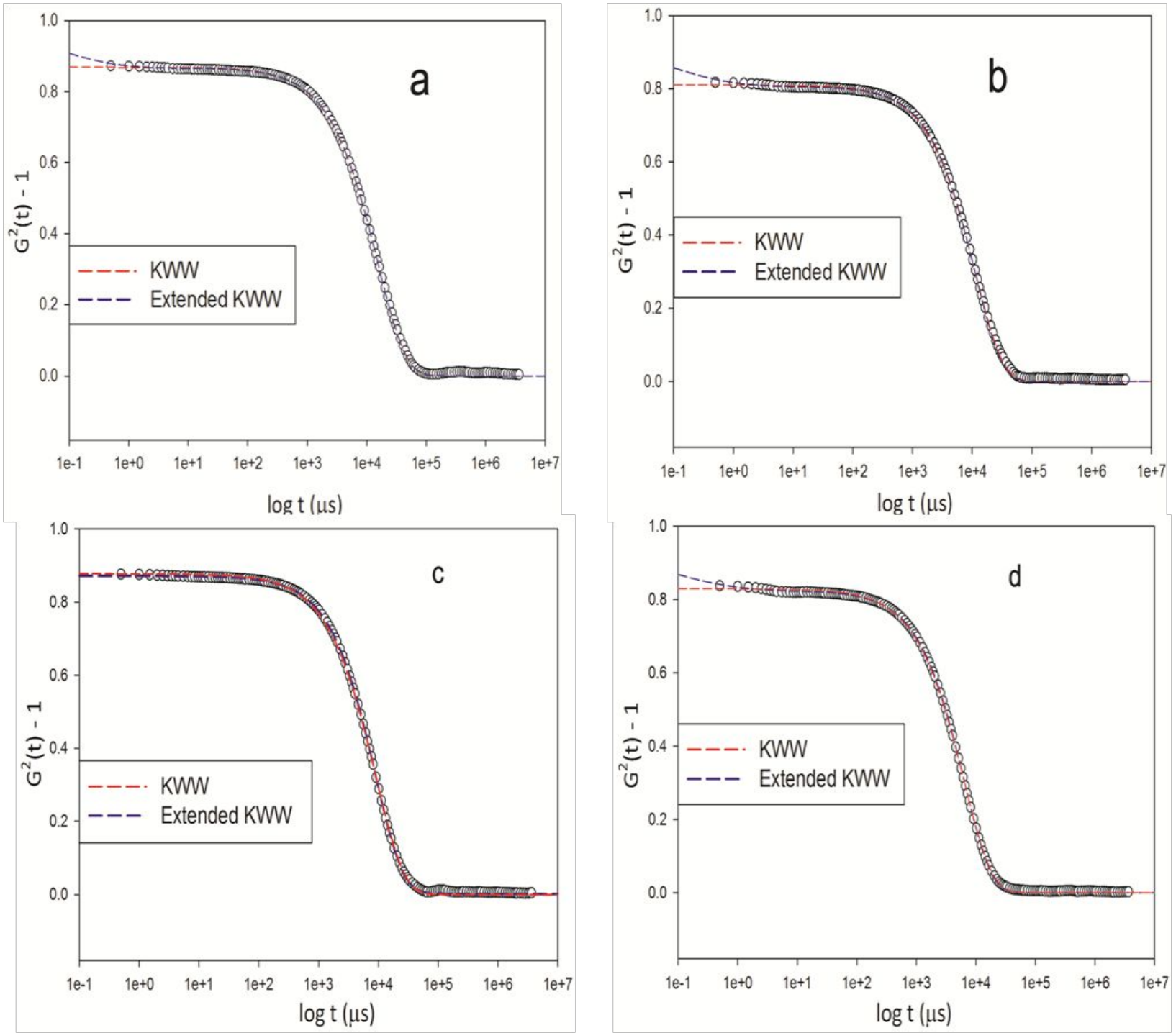

Figure S2. Normalized $\mathrm{G}^{2}(\mathrm{t})-1$ ICFs of $\left[\mathrm{C}_{2} \mathrm{mim}\right][\mathrm{OTf}]_{0.5}\left[\mathrm{NTf}_{2}\right]_{0.5}$ fitted by KWW and extended KWW approximations at (a) $303 \mathrm{~K}$, (b) $313 \mathrm{~K}$, (c) $323 \mathrm{~K}$ and (d) $333 \mathrm{~K}$ 

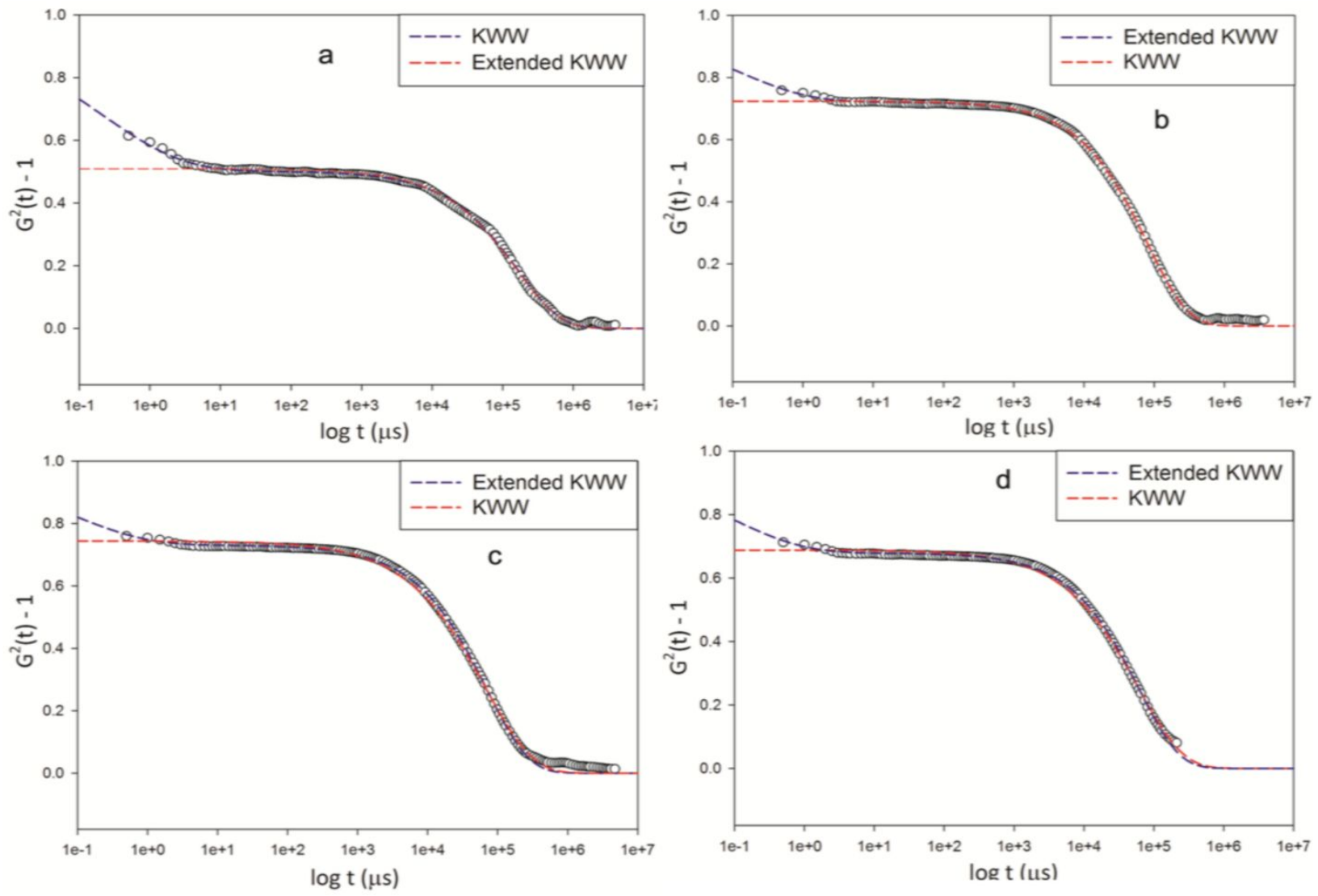

Figure S3. Normalized $\mathrm{G}^{2}(\mathrm{t})-1$ ICFs of $\left[\mathrm{C}_{2} \mathrm{mim}\right]\left[\mathrm{BF}_{4}\right]_{0.5}\left[\mathrm{NTf}_{2}\right]_{0.5}$ fitted by $\mathrm{KWW}$ and extended KWW approximations at (a) $303 \mathrm{~K}$, (b) $313 \mathrm{~K}$, (c) $323 \mathrm{~K}$ and (d) $333 \mathrm{~K}$ 

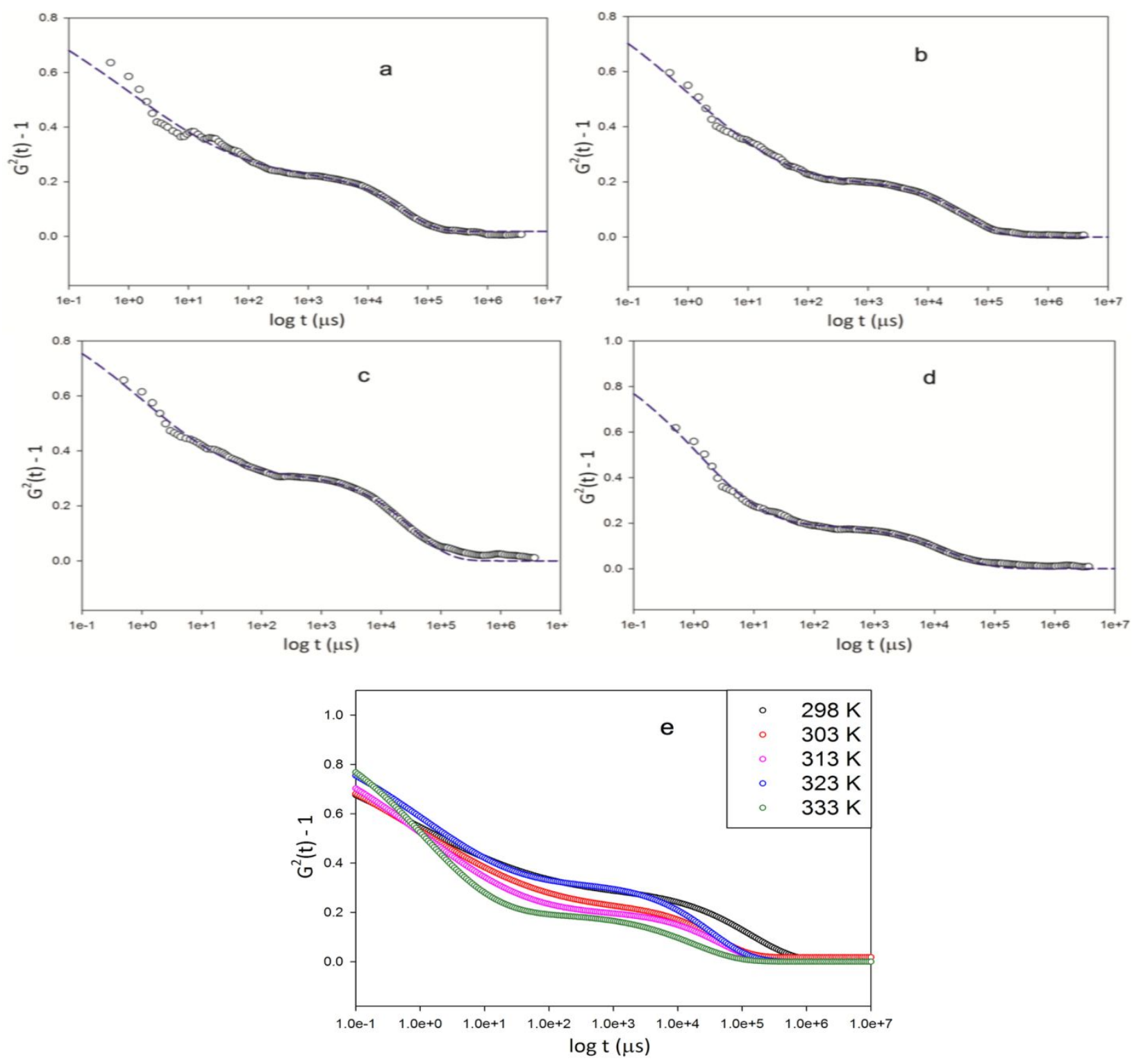

Figure S4. Normalized $\mathrm{G}^{2}(\mathrm{t})-1$ ICFs of $\left[\mathrm{C}_{2} \mathrm{mim}\right]\left[\mathrm{MeSO}_{3}\right]_{0.5}\left[\mathrm{NTf}_{2}\right]_{0.5}$ fitted by KWW and extended KWW approximations at (a) $303 \mathrm{~K}$, (b) $313 \mathrm{~K}$, (c) $323 \mathrm{~K}$ and (d) $333 \mathrm{~K}$ and (e) deconvoluted ICFs at $2303-333 \mathrm{~K}$ of $\left[\mathrm{C}_{2} \mathrm{mim}\right]\left[\mathrm{MeSO}_{3}\right]_{0.5}\left[\mathrm{NTf}_{2}\right]_{0.5}$ 


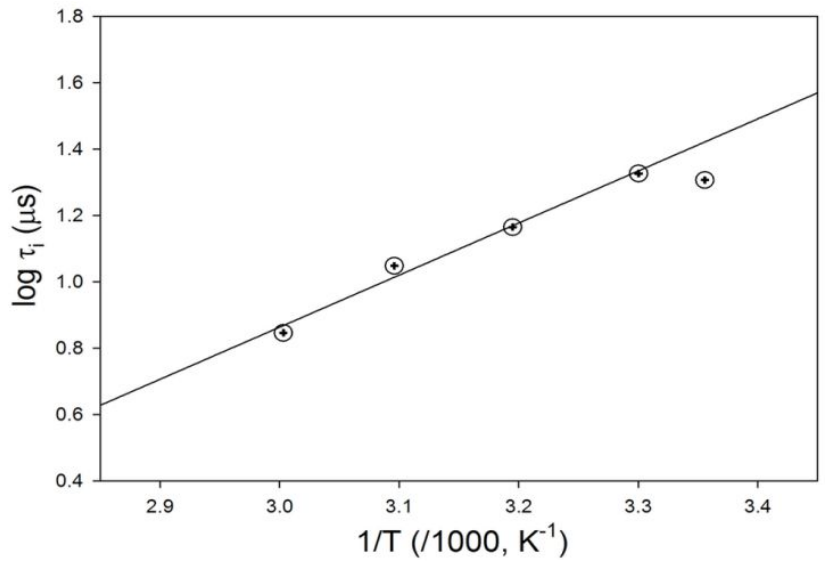

Figure S5. Logarithm of the intermediate relaxation times against inverse temperature for $\left[\mathrm{C}_{2} \mathrm{mim}\right]\left[\mathrm{MeSO}_{3}\right]_{0.5}\left[\mathrm{NTf}_{2}\right]_{0.5}$
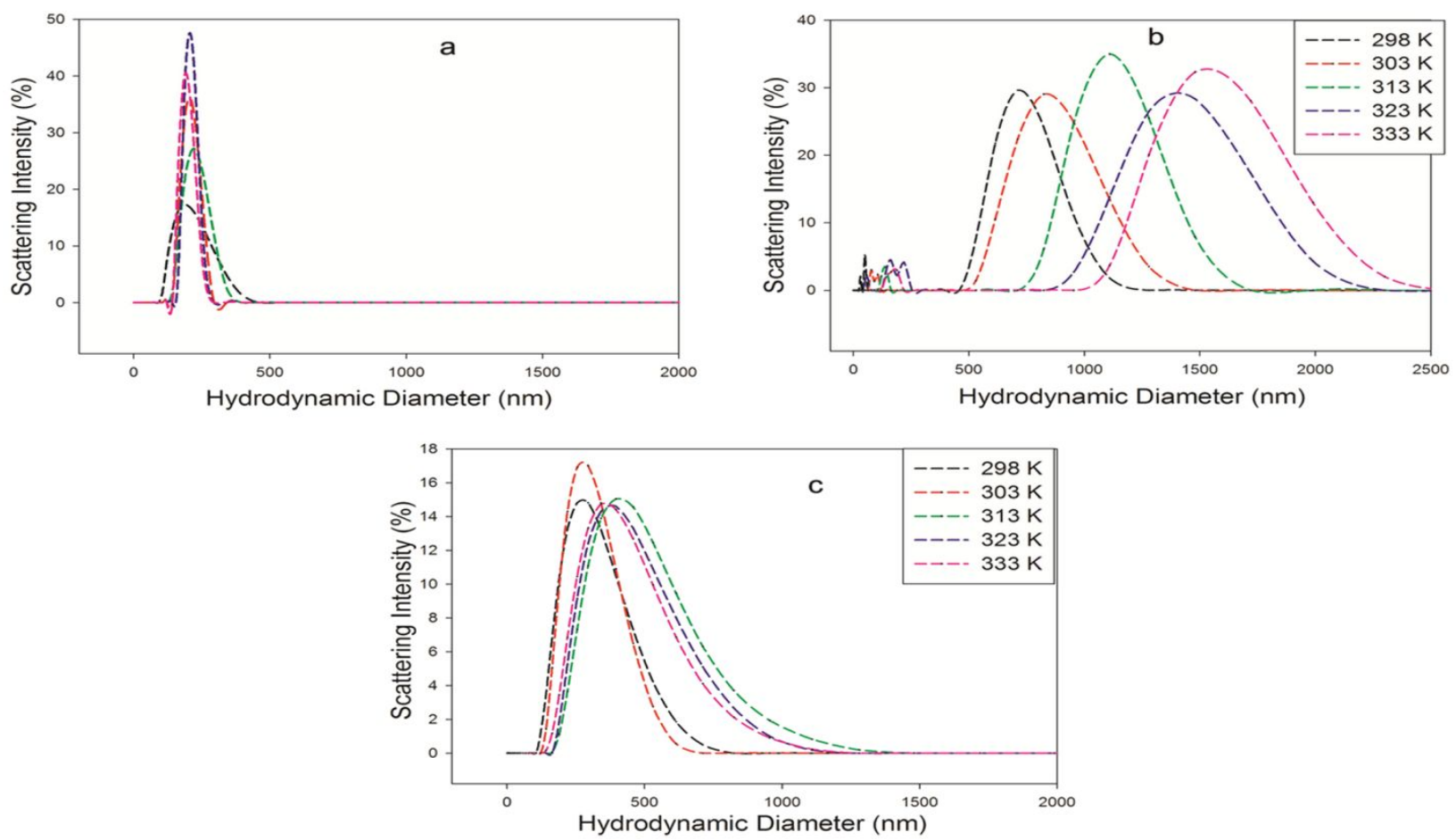

Figure S6. Intensity distribution of hydrodynamic diameter of mesoscale clusters in (a) $\left[\mathrm{C}_{2} \mathrm{mim}\right][\mathrm{OTf}]_{0.5}\left[\mathrm{NTf}_{2}\right]_{0.5}$, (b) $\left[\mathrm{C}_{2} \mathrm{mim}\right]\left[\mathrm{BF}_{4}\right]_{0.5}\left[\mathrm{NTf}_{2}\right]_{0.5}$ and (c) $\left[\mathrm{C}_{2} \mathrm{mim}\right]\left[\mathrm{MeSO}_{3}\right]_{0.5}\left[\mathrm{NTf}_{2}\right]_{0.5}$ 


\section{S.2. Alternatives to Microfiltration: The Issue of Avoiding Dust during the DLS Measurements}

Dust is a very important concern during the performance of DLS experiments. We have been fully aware of the interference of the dust in our samples from the beginning of the experiment. The commonplace practice is to use a microfilter in order to pass the sample to the cuvette for carrying out DLS experiments. However, passing viscous ILs and DSILs through microfilters was indeed not easy; often, we experienced the problem of foaming while passing the DSILs through $0.2 \mu \mathrm{m}$ Telfon filters; formation of bubbles could not be avoided at times as well. Our objective turned in to find a route in order to avoid the use of filters - while reproducing the data obtained for the samples passed through the filters. Thus we used a $100 \mu 1$ micropipette and disposable universal micropipette-tips to transfer the DSILs into the cuvette. In each case, first few drops of the samples were discarded and then the samples were taken into the cuvette. Immediately after that, the cuvette was sealed with a Teflon stopper and subjected for experiments. Also, immediately before each of the experiments the glass cuvette was thoroughly cleaned and washed by means of deionized water, absolute ethanol and acetone and dried at 110 ${ }^{\circ} \mathrm{C}$ (similar precautionary measures were taken for the glassware, vials and graduated glasspipettes for example, prior to the preparation of the DSILs as well). We also made certain that all glassware and disposable equipments were kept inside air-tight dry plastic containers all the time- so the contact period of the apparatus with air would be as minimal as possible. We compared the two sets of correlograms for the DSILs - one for which we filtered the samples and the other for which where we did not - and observed that the ultraslow relaxation events for the latter case could be retrieved as nearly similar to the former case. Similar comparison could be drawn when we obtained the correlograms for the pure ILs as well. Below we show an example - in Figure S7(a) and S7(b), where two normalized correlograms for $\left[\mathrm{C}_{2} \mathrm{mim}\right]\left[\mathrm{BF}_{4}\right]_{0.5}\left[\mathrm{NTf}_{2}\right]_{0.5}$ at $298 \mathrm{~K}$ are shown. The $\mathrm{G}^{2}(\mathrm{t})-1$ scattering function for the filtered DSIL is less intense compared to the non-filtered one - which is due to the insufficient settling time of the DSILs (2 hours) after the pass-through off the filters. The ultralsow relaxation time for the filtered sample appears to be $6.78 \times 10^{5} \mu \mathrm{s}$, which is fairly close to the relaxation time for the non-filtered sample $\left(6.20 \times 10^{5} \mu \mathrm{s}\right)$ when fitted to the extended KWW function. Size distribution of the mesoscopic structures is also nearly similar for both of filtered and nonfiltered samples (Figure S7(c)). This ensures that the results are not getting hampered even when we are not filtering the samples - provided the cleaning protocol has been followed thoroughly before the preparation of DSILs and incorporation of the samples into the cuvette. This trend was commonplace for all other DSILs and pure ILs as well. Thus, we have considered the results obtained from the non-filtered samples in the long run and showed evidence that the relaxation profiles are not due to the interference of dust particles. 

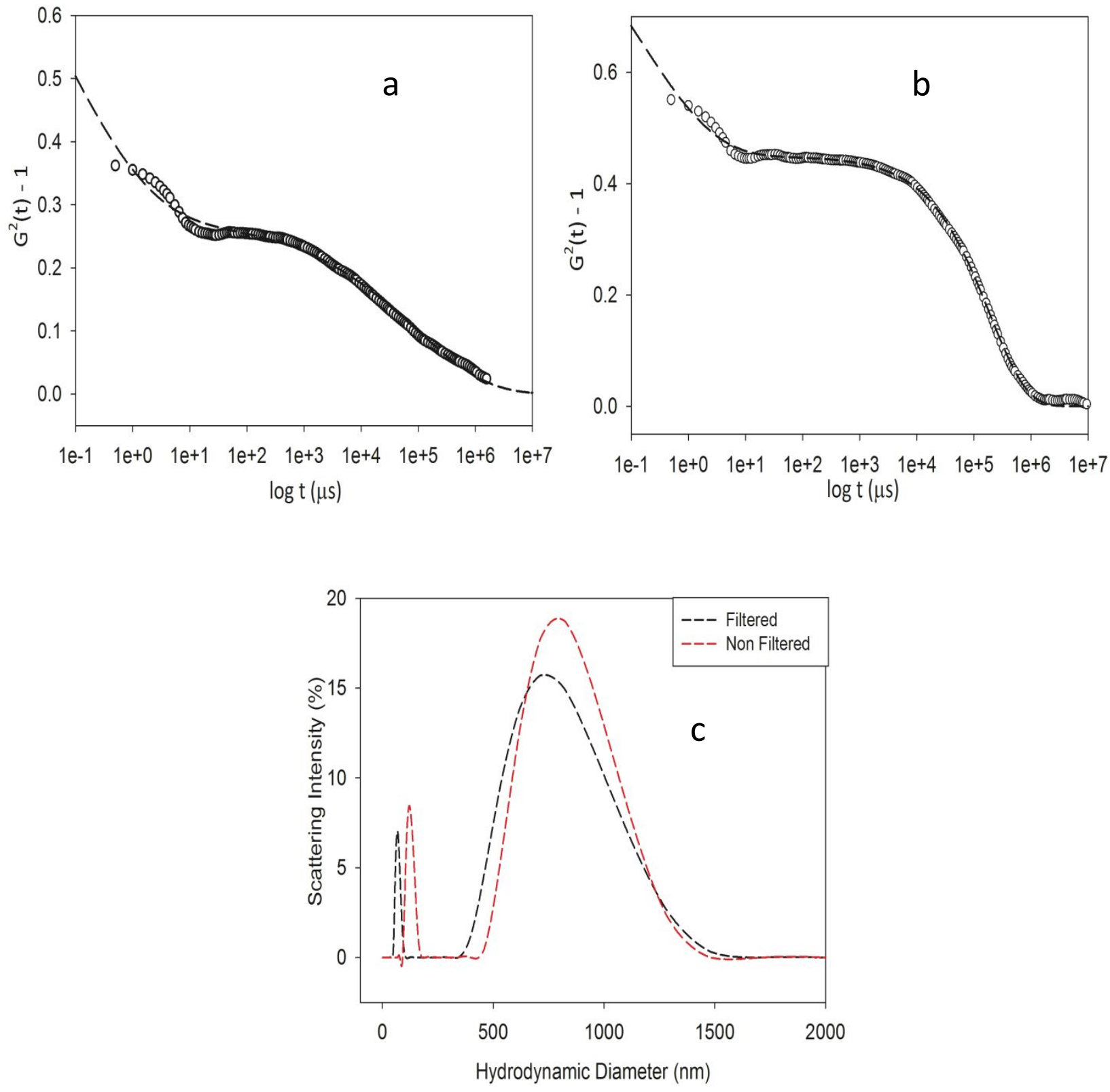

Figure S7. (a) Normalized second-order correlation functions of filtered

$\left[\mathrm{C}_{2} \mathrm{mim}\right]\left[\mathrm{BF}_{4}\right]_{0.5}\left[\mathrm{NTf}_{2}\right]_{0.5}$ at $298 \mathrm{~K}$ (settling time: $2 \mathrm{hrs}$ ), (b) correlation function of non-filtered $\left[\mathrm{C}_{2} \mathrm{mim}\right]\left[\mathrm{BF}_{4}\right]_{0.5}\left[\mathrm{NTf}_{2}\right]_{0.5}$, (c) scattering intensity of size distribution for $\left[\mathrm{C}_{2} \mathrm{mim}\right]\left[\mathrm{BF}_{4}\right]_{0.5}\left[\mathrm{NTf}_{2}\right]_{0.5}$ at $298 \mathrm{~K}$. 